Meta

Journal des tradlucteurs

Translators' Journal

\title{
Réflexion sur une expérience de traduction occasionnelle
}

\section{Marie-Noëlle}

Volume 13, numéro 3, septembre 1968

URI : https://id.erudit.org/iderudit/003742ar

DOI : https://doi.org/10.7202/003742ar

Aller au sommaire du numéro

Éditeur(s)

Les Presses de l'Université de Montréal

ISSN

0026-0452 (imprimé)

1492-1421 (numérique)

Découvrir la revue

Citer cet article

Marie-Noëlle (1968). Réflexion sur une expérience de traduction occasionnelle. Meta, 13(3), 116-123. https://doi.org/10.7202/003742ar d'utilisation que vous pouvez consulter en ligne.

https://apropos.erudit.org/fr/usagers/politique-dutilisation/ 


\section{SUR UNE EXPÉRIENCE}

\section{DE TRADUCTION DCCASIONNELLE}

\section{INTRODUCTION}

C'est à une épreuve bien étrange que se trouve convié un traducteur occasionnel, appelé à réfléchir sur son expérience pour en rendre compte à un groupe de traducteurs professionnels.

J'avouerai franchement n'avoir jamais jusqu'ici fait semblable travail, n'ayant été conduite à traduire des textes, généralement courts: articles de revue, passages de lecture, poésies ou prières, que dans le but d'initier le lecteur à un domaine qui lui est étranger; l'œcuménisme en particulier, le judaïsme ou les rapports judéochrétiens.

Pour répondre à votre attente j'ai cependant amorcé une réflexion, que je m'excuse de présenter de façon nécessairement très personnelle, car c'est mon expérience dont j'essaie de rendre compte, et elle est conditionnée par un ensemble de circonstances, tempérament, milieu familial et scolaire, études faites; les mille événements qui ont jalonné mon cheminement dans l'existence.

\section{PRÉPARATION LOINTAINE}

Deux atouts dans mon jeu:

a) une familiarité dès l'enfance, avec plusieurs langues étrangères, a pu m'aider à dissocier spontanément le concept et les mots ou expressions dans lesquels il s'incarne, a pu également créer une certaine exigence d'expression correcte, l'oreille y étant sensibilisée comme d'instinct.

b) une formation scientifique - L'étude des mathématiques ayant engendré un besoin de lucidité dans la pensée et de rigueur dans l'expression, tandis que la méthode des sciences expérimentales m'initiait à la soumission au réel, au fait tel qu'il est et non tel que je l'ai conçu ou prévu. Qualités qui peuvent jouer un rôle important dans la traduction telle que je l'envisage.

\section{CE QUI PARATT REQUTS}

D'UN BON TRADUCTEUR

- Posséder à fond la langue dans laquelle il traduit, la maîtriser suffisamment pour être en mesure de s'essayer à rendre une phrase difficile de plusieurs façons, 
jusqu'à ce que satisfaction s'ensuive et que le lecteur éventuel n'ait pas l'impression désagréable qu'il s'agit d'une traduction.

- Avoir avec la langue de l'original une familiarité suffisante, sans qu'il soit pour cela nécessaire de la posséder parfaitement.

- Se mouvoir enfin, avec aisance, dans le domaine propre qui constitue l'objet de la traduction.

Je voudrais établir ici une gradation:

- S'il s'agit d'un texte non spécialisé, une solide culture générale me semble pouvoir suffire pour une traduction honnête.

- S'il s'agit d'un texte appartezant à un domaine technique: sciences physiques, médecine, droit, musique, etc., il va de soi qu'un non-professionnel ne saurait s'y aventurer car il ignore le « jargon » propre à ce secteur et risque de commettre des erreurs de vocabulaire grossières.

- S'il s'agit maintenant d'un texte où s'exprime l'âme d'un peuple, d'un groupe humain, d'un homme, une qualité supplémentaire me paraît requise du traducteur; une certaine «connaturalité » avec le sujet en même temps qu'une connaissance approfondie de la langue, une sympathie qui lui permette de vibrer avec l'auteur, de s'identifier pour une part avec lui, de regarder avec ses yeux, de comprendre avec son cœur. En particulier s'il est question de traduire un poème, une prière, un livre traitant du folklore, ou de la religion d'un groupe humain, une technique de la traduction même parfaite me paraît absolument insuffisante.

Présenter un texte correct, impeccable sur le plan du vocabulaire, de la grammaire, ne suffit pas, car il s'agit d'introduire le lecteur dans une mentalité qui lui est étrangère, l'amener à vibrer à une expérience humaine, lui faire découvrir les harmoniques d'une musique propre, que le traducteur a dû transposer en une autre clé pour la lui rendre accessible.

\section{TRADUCTION DE L'ANGLAIS AU FRANÇAIS}

Le génie comme la langue de la France et de l'Angleterre ont souvent été mis en contraste. Je mentionnerai simplement quelques nuances qui mont frappée davantage:

En anglais, me semble-t-il, les phrases sont généralement plus courtes, plus juxtaposées, moins construites qu'en français, avec un usage plus réduit des particules de subordination.

À titre d'exemple, voici quelques lignes relevées au cours d'une lecture faite récemment:

The speakers at the Toronto Congress took for granted that the Church is in constant need of renewal and reform./ This in itself is quite remarkable./ We have here one of the dramatic effects of Vatican II Council./ Hardly a. paper was concerned with reform as an ecclesiological problem./ The lecture with the promising title «Institution and Charisms», delivered by Bishop Butler, did not really deal with the issue./ The Bishop was curiously cautious./ He warned his listeners...

En français, je proposerais la traduction suivante:

Les conférenciers du Congrès de Toronto tenaient pour assuré que 1'Église est en constant besoin de réforme et de renouveau, chose en soi très remarquable et l'un des effets spectaculaires du Concile Vatican II./ C'est tout 
juste si un rapport envisagea la réforme comme un problème ecclésiologique, et encore, la conférence au titre prometteur de Dom Butler «Institution et charismes » n'a-t-elle pas vraiment traité du sujet./ L'évêque se montra étrangement circonspect, mettant en garde ses auditeurs...

Le style anglais, par ailleurs, me paraît moins imagé, quoique plus concret que le français. Ainsi le thème de l'Expo 67 «Terre des Hommes » a été traduit «Man and his World» expression qui est pour moi, Française, moins évocatrice, moins parlante que celle de Saint-Exupéry.

Autre exemple: comment rendre en anglais les mots «l'optique du catholicisme »? Peut-être par un equivalent comme « according to the viewpoint of the Roman Catholic Church », expression plus concrète, moins concise, moins imagée.

Dans le livre du rabbin Stuart Rosenberg, sur le judaïsme, se trouve un passage emprunté à Daniel-Rops, le paragraphe suivant que j'en extrais me semble caractéristique de ce que j'essaie de rendre:

Texte français: «Lorsqu'il se lève pour porter son message, dans quel cadre, avec quels collaborateurs Jésus agit-il ? »

Texte anglais: "When he began his ministry, in what context did he do so, and who were his helpers, his collaborators?»

En anglais les répétitions fréquentes d'un même mot ne semblent pas répugner, alors que le français s'ingéniera à varier l'expression, à employer un synonyme, un équivalent. Ainsi du passage suivant:

... Only Israel was to be different, and the Covenant was the sign of its uniqueness - chosen to serve as the lone monotheist among the nations. Now however the Phophets endow Israel's covenant with a missionary meaning: Israel must persevere until the End of Days, when God will make himself known to all the nations, as he did to Israel. If anything, Israel's religious burden... ${ }^{1}$

Voici maintenant la traduction que j'ai esquissée:

Israël seul était différent, et l'Alliance était le signe de la singularité du peuple, choisi pour être parmi les nations le seul à honorer le Dieu unique. Maintenant cependant les Prophètes revêtaient d'une signification missionnaire l'alliance conclue avec Israël. Celui-ci doit se perpêtuer jusqu'à la fin des temps, époque où Dieu se fera connaître lui-même aux nations comme il l'avait fait au peuple choisi. Le résultat, c'est que le fardeau religieux de ce peuple...

Je dirais encore que parfois l'anglais me paraît peu logique. Ainsi l'expression Faithfully yours, employée me dit-on dans le langage des affaires, en Angleterre, lorsqu'on s'adresse à des inconnus, me semble étrange. Comment peut-on assurer de sa fidélité quelqu'un qu'on ne connaît pas?

\section{TRADUCTION D'UN LIVRE}

SUR LA RELIGION JUIVE

C'est la première fois que j'abordais la traduction d'un livre, et peut-être ne m'y serais-je pas risquée si on ne me l'avait demandé et si je n'avais eu la persuasion qu'il n'existe aucun livre en français accessible à la masse et présentant l'ensemble de la religion et de la vie juives.

La tâche se trouvait d'ailleurs simplifiée du fait que l'auteur du livre Judaism, le rabbin Stuart Rosenberg, de Toronto, s'adressant précisément à un public

1. Stuart Rosenberg, Judaism, Glen Rock (N.J.), Paulist Press, 1966, p. 25. 
chrétien, avait pris soin de s'exprimer en une langue accessible à celui-ci, apportant les explications requises partout où cela semblait nécessaire, donnant la traduction anglaise des termes hébreux employés, munissant même son volume d'un glossaire. Toutes choses qui rendaient plus aisée la tâche du traducteur.

J'ajouterai qu'une longue familiarité avec le judaïsme, aussi bien dans son expression anglaise que dans son expression française, mon initiation à la langue hébraïque étudiée plusieurs années durant, étaient des appoints qui m'autorisaient à me lancer dans l'entreprise. S'il s'était agi de présenter l'islam ou une religion de l'Orient, je me serais évidemment récusée, faute de compétence, de même s'il avait été question de présenter un livre sur la mystique juive, la philosophie juive du Moyen Âge, ou autre sujet spécialisé, accessible seulement à un professionnel.

Les difficultés rencontrées n'ont donc consisté, de ce point de vue, qu'en nuances subtiles qu'un traducteur ignorant du judaïsme aurait risqué de commettre. Ainsi le terme bar-mitzvah, s'il est précédé de l'article masculin, signifie le garçon qui vient d'accomplir la cérémonie par laquelle il est devenu «fils du précepte », c'est-à-dire adulte sur le plan religieux et désormais soumis à l'observance de la loi mosaïque. Le même terme, précédé de l'article féminin, désigne la cérémonie elle-même. Or l'article anglais the n'a pas de masculin et de féminin. Quel article donc employer dans la traduction française?

Autre exemple: «the fall holiday of Succoth», pourrait à priori se traduire par « les vacances automnales de Souccoth», ou encore «le festival d'automne de Souccoth »; c'est la dernière traduction qui est la bonne. Mais ici le contexte déterminera aisément le choix de la traduction correcte.

Je voudrais signaler ici le bénéfice retiré de la traduction d'un ouvrage religieux. À travers bien des passages, parallèles implicites, références occasionnelles, que peut-être le lecteur ne relèvera pas, mais auxquels le traducteur sera plus sensible, j'ai une fois de plus constaté combien le représentant d'une religion étrangère, même très bien disposé, même croyant bien connaître l'objet de ses références, a tendance à durcir, à simplifier à outrance, à stéréotyper la religion qu'il prend pour terme de comparaison. Sans doute la connaît-il jusqu'à un certain point, mais comme on sent que c'est de l'extérieur, souvent dans un revêtement linguistique d'époques antérieures, en tout cas à travers une optique qui l'empêche de saisir les harmoniques dont sont chargés des termes usuels comme celui de «sacrement», ou la conception du «salut par la foi », etc.

Pour un traducteur, intéressé par ailleurs au dialogue religieux, il me semble que pareille constatation est enrichissante à divers points de vue, et peut éventuellement constituer une amorce de dialogue...

\section{TRANSCRIPTION DES TERMES HÉBREUX}

Pour le traducteur occasionnel qui ignore l'hébreu, la question de la transcription en français de termes hébraïques épars dans un texte anglais ne se serait pas posée. Il lui aurait suffi de copier les mots tels qu'ils se présentaient dans l'original anglais. Pour qui a étudié l'hébreu, à travers la transcription anglaise, c'est le mot hébreu qui apparaîtra spontanément, d'où un petit problème. Une transcription dans une langue étrangère présente toujours des difficultés et ne 
rend l'original que de façon insatisfaisante, souvent approximative; parfois aucun équivalent réel ne se présente.

Fallait-il, s'adressant à des lecteurs francophones, conserver la transcription anglaise ? N'était-il pas plus naturel de rendre les mots hébreux par un équivalent phonétique emprunté à l'orthographe française usuelle? Quelques exemples vont préciser le problème et fournir l'occasion d'une réponse:

- le mot hébreu $K-C H-R$ (3 lettres) qui signifie: pur, est rendu en anglais par Kosher.

- le même mot, en France, est habituellement orthographié cachère.

Quelle orthographe adopter? Spontanément la deuxième vient à l'esprit pour qui écrit à l'usage de francophones. Mais nous sommes au Québec, en Amérique du Nord, le mot en question figure partout: publicité, boîtes de conserves, enseignes de boutiques... il est devenu familier dans sa transcription anglaise à tous, sans distinction de langue. L'écrire différemment n'était-ce pas embrouiller les esprits? J'ai opté sans hésitation pour la transcription anglaise, familière ici. En France j'aurais agi différemment. Une deuxième remarque peut être faite à l'occasion de ce même mot. La vocalisation kôsher au lieu de cachère, se réfère à la différence de prononciation de l'hébreu chez les juifs Askenazes (Allemagne, Europe orientale) et Sepharades (Espagne, Bassin méditerranéen), la même voyelle ayant la valeur de $o$ pour les premiers, et suivant les cas mais très ordinairement, de $a$ pour les seconds.

Autre mot qui m'a fait hésiter: B'nai $B^{\prime}$ rith (étymologiquement: fils de l'Alliance). La transcription anglaise est à première vue étrange pour un Français de France qui a étudié l'hébreu; il écrirait plutôt: Benê Berith. Fallait-il transcrire ainsi ? Oui si l'on écrit pour la France, mais en France l'association caritative des B'nai B'rith est relativement peu connue; en Amérique du Nord, en revanche, elle est extrêmement répandue et la transcription anglaise est familière à l'homme de la rue. Là encore il fallait la conserver.

Remarque analogue pour le mot hébreu qui signifie «paix»: Shalom en anglais, et plutôt Chalome si l'on se réfère à la prononciation française commune.

Un problème se pose également pour la transcription des sons consonantiques ou vocaliques qui n'ont pas d'équivalent dans la langue où l'on traduit: ainsi du son allemand ch (dur) qui en hébreu est celui de la lettre caph en certains cas. Cette lettre ainsi que plusieurs autres dénommées "begadkephat 》 (barbarisme destiné à aider la mémoire) a en effet deux prononciations, suivant les cas régis par la grammaire. Le rabbin Rosenberg, dans son livre, a rendu ce son d'ordinaire par le $k$ (omettant la question de la double prononciation); j'ai opté pour le $k h$. Souvent ce même son est rendu par $c h$ même en Amérique; en français cela eût prêté à confusion.

Bien des remarques analogues pourraient être faites simplement à l'occasion des mots usuels chez les juifs, même ceux qui n'ont pas étudié l'hébreu. En ce qui me concerne, j'ai résolument simplifié la question en adoptant une fois pour toutes, sauf très rares exceptions, la transcription anglaise usuelle, employée par le rabbin et que de nombreux lecteurs, désireux de s'informer sur le judaïsme et connaissant la langue anglaise, rencontreront fréquemment. La seule exception 
notable est le remplacement du $u$ par ou chaque fois où le français le demande normalement: ainsi kiddush a été rendu par kiddoush.

Je signale pour terminer l'usage du $k$ (familier à la langue anglaise) à la fois pour les lettres distinctes caph et coph, même si la logique, du moins pour un Français, eût demandé de distinguer, et même si le $k$ n'est guère utilisé dans la langue française. C'est le désir de ne pas dérouter les lecteurs qui a présidé au choix., comme il a été expliqué plus haut.

\section{LES HANDICAPS DU TRADUCTEUR OCCASIONNEL}

Je ne mets pas en doute que l'absence de formation à la technique de la traduction ne représente un handicap: perte de temps à chercher, longtemps parfois, l'expression adéquate d'une formule intraduisible telle quelle, et que le recours à plusieurs dictionnaires n'arrive pas à élucider... Risque aussi de méprise: le traducteur croyant avoir compris un mot de l'original, apparemment le même que dans sa langue (mots anglais et français qui, identiques à l'origine, ont évolué différemment), et auquel inconsciemment il donne cette dernière valeur alors qu'elle en a une autre dans l'original: ainsi actual, rendu par « actuel » alors qu'il signifie réel, effectif; prejudice rendu par «préjudice» alors qu'il signifie préjugé, etc. Danger peut-être aussi, dans la traduction de l'anglais, de laisser subsister des anglicismes, assez couramment utilisés en français mais incorrects: traduire I realized par « j'ai réalisé ». Hésitation parfois sur le sens à donner à un terme, ou sur la manière de le rendre de façon adéquate lorsqu'il n'a pas d'équivalent en français: ainsi le mot scholar m'a souvent causé des diffícultés.

De toute façon, la prudence devrait engager le traducteur d'occasion à soumettre sa traduction à la révision d'un professionnel, ou, à tout le moins, recourir à lui pour les passages qui font difficulté. Cela surtout s'il s'agit d'un ouvrage important ou dont la pensée est nuancée. Il y a là un acte d'honnêteté vis-à-vis du lecteur et une garantie de qualité dans le travail.

Il me paraît néanmoins que, pour comporter le maximum de bénéfice, semblable démarche devra se faire seulement après que le traducteur occasionnel se sera colleté avec la difficulté, aura remis plusieurs fois sur le métier son essai de traduction pour essayer d'en venir à bout lui-même. Pour celui qui a reçu une formation scientifique, ce sera, je pense, la démarche spontanée de l'esprit. Traduire, n'est-ce pas un peu comme résoudre un problème, venir à bout d'un obstacle? N'est-ce pas aussi se soumettre à une exigence du réel qui, loin de porter à la passivité, doit stimuler la réflexion, l'ingéniosité, la faculté d'invention?

Le recours au professionnel apportera alors infiniment plus que si on l'avait consulté aussitôt qu'on s'est heurté à la difficulté.

L'exercice de la traduction, pour celui qui s'y adonne occasionnellement et n'en fait pas son métier, m'apparaît comme une discipline formatrice de l'esprit, en même temps qu'une invitation à acquérir une maîtrise plus grande dans un travail dont il aura perçu, d'expérience personnelle, les exigences et la complexité, je voudrais ajouter aussi la joie, joie de réussir, de moins en moins mal, à rendre accessible au lecteur, dans la plénitude de sa teneur, la pensée qu'on désire lui transmettre. 


\section{PETIT GLOSSAIRE DE TERMES HÉBRAÏQUES ENTRÉS DANS LE VOCABULAIRE UNIVERSEL}

Alleluia et Amen, deux mots tellement utilisés dans la liturgie des Églises chrétiennes que beaucoup de gens en ignorent l'origine hébraïque.

Il peut être intéressant pour des traducteurs de savoir que le premier est un impératif, deuxième personne du pluriel du verbe $H L L$, et signifie exactement: «Louez Yah» ou «Louez Dieu» (Yah étant un des noms bibliques de Dieu).

Amen provient d'une racine signifiant à la fois: fidélité et vérité. C'est dire la richesse de ce terme, rendu bien imparfaitement par le français: "Ainsi soit-il ».

Hosannah. Encore un mot de notre liturgie, qui en a simplifié le son et l'orthographe. En hébreu c'est Hôshiah-na = Sauve-donc! Il est intéressant de noter à ce sujet que l'usage chrétien a modifié le sens primitif du terme, transformant en acclamation triomphale une invocation de caractère implorant.

Jéhovah ou Yahvé? Le tétragramme sacré $Y H W H$, nom intime de Dieu en quelque sorte, que la Bible présente comme révélé à Moïse au Buisson ardent (Ex. 3, 15), est devenu dans la tradition juive de très bonne heure un nom si saint qu'il était interdit à l'homme de le prononcer, sinon au grand-prêtre, au saint des saints du temple de Jérusalem, une fois l'an, au grand jour des Expiations. De ce fait sa prononciation exacte s'est perdue au cours des temps. Les philologues chrétiens se basant sur une connaissance de l'évolution de la langue hébraïque ont proposé la transcription Yahvé ou Yahweh, comme la plus probable. Les juifs pieux ne se permettent pas de prononcer ce nom et y suppléent par des équivalents dont le plus courant est Adonaï qui signifie «Seigneur». C'est précisément pour indiquer au lecteur du texte biblique qu'il faut prononcer Adonaï là où le tétragramme se trouve écrit que les massorètes (savants hébreux du vII ${ }^{e}$ siècle de notre ère) ont placé sous les consonnes du tétragramme (on sait que les langues sémitiques n'ont pas de voyelles) le système de points et de tirets (qui suppléent aux voyelles) du mot Adonaï. Des chrétiens peu savants dans la langue hébraïque et qui ne connaissaient que le texte biblique massorétique - avec les pointsvoyelles - ont spontanément prononcé le mot tel qu'il se trouvait écrit: Jéhovah. Véritable barbarisme puisqu'il s'agit des consonnes d'un mot avec les voyelles d'un autre. Cette appellation fautive n'est plus guère employée, à tout le moins, par ceux qui sont familiers des traductions modernes de l'Écriture.

Talmud: Ensemble de la Halakhah et de la Haggadah, commentaires légaux et non légaux échafaudés sur la Mishnah au cours des $\mathrm{III}^{\mathrm{e}}$, IV et $\mathrm{V}^{\mathrm{e}}$ siècles de notre ère. La Mishnah elle-même ayant été la première compilation de la tradition orale de l'Écriture, élaborée au cours de plusieurs siècles et mise par écrit aux alentours de l'an 200. Il existe en réalité deux Talmuds, l'un composé à Babylone, l'autre en Palestine. Lorsqu'on parle du Talmud tout court, c'est toujours du premier qu'il s'agit. Ce terme qui excite tant d'appréhension chez de nombreux chrétiens, dérive de la racine trilittère $L M D$, d'où viennent également des mots du vocabulaire courant comme talmid (élève), molammed (instituteur). Le Talmud n'est autre que l'ensemble de l'enseignement traditionnel juif, du moins la majeure partie de celui-ci, car la tradition orale le déborde notablement. 
Torch: Au sens strict, signifie le Pentateuque, les cinq livres de Moïse, les cinq premiers livres de la Bible. Au sens large, il est souvent employé pour désigner la Bible hébraïque en son entier, et la Torah orale n'est autre que l'ensemble de la tradition orale.

Synagogue: Bien des chrétiens imaginent que c'est un mot hébreu. Pour qui a fait un peu de grec il est évident que ce terme qui désigne l'assemblée religieuse juive, ou le lieu où elle se rassemble (cf. notre ecclesia = église), est en réalité un terme grec, issu de l'usage du judaïsme hellénique.

Kabbale ou Cabbale: Est un mot qui signifie tradition. De même que le mot français se réfère au latin tradere $=$ transmettre, le mot hébreu dérive de la racine $Q B L$ qui a le même sens de transmission. Avec le temps le terme de Cabbale en est venu à désigner proprement l'ensemble de la mystique ésotérique juive. Son livre principal est le Zohar (de la racine $Z H R=$ briller) ou Livre de la Splendeur.

Sœur MARIE-NoËLle, n.d.s. 